\title{
gु \\ Antiferromagnetic order in MnO spherical nanoparticles
}

\author{
C. H. Wang, ${ }^{1}$ S. N. Baker, ${ }^{1, *}$ M. D. Lumsden, ${ }^{1}$ S. E. Nagler, ${ }^{1}$ W. T. Heller, ${ }^{1}$ G. A. Baker,,${ }^{1, \dagger}$ P. D. Deen, ${ }^{2, \dagger}$ \\ L. M. D. Cranswick, ${ }^{3}$ Y. Su, ${ }^{4}$ and A. D. Christianson ${ }^{1}$ \\ ${ }^{1}$ Oak Ridge National Laboratory, Oak Ridge, Tennessee 37831, USA \\ ${ }^{2}$ Institut-Laue Langevin, 6 Rue Jules Horowitz, F-38042 Grenoble, Cedex 9, France \\ ${ }^{3}$ Canadian Neutron Beam Centre, National Research Council of Canada, Chalk River Laboratories, Chalk River, Ontario, Canada K0J $1 \mathrm{JO}$ \\ ${ }^{4}$ Jülich Centre for Neutron Science, Forschungszentrum Jülich, Outstation at FRM II, Lichtenbergstrasse 1, D-85747 Garching, Germany
}

(Received 29 March 2011; published 15 June 2011)

\begin{abstract}
We have performed unpolarized and polarized neutron diffraction experiments on monodisperse 8- and 13-nm antiferromagnetic $\mathrm{MnO}$ nanoparticles. For the 8-nm sample, the antiferromagnetic transition temperature $T_{N}$ $(114 \mathrm{~K})$ is suppressed compared to that in the bulk material $(119 \mathrm{~K})$, while for the $13-\mathrm{nm}$ sample $T_{N}(120 \mathrm{~K})$ is comparable to that in the bulk. The neutron diffraction data of the nanoparticles is well described using the bulk MnO magnetic structure but with a substantially reduced average magnetic moment of $4.2 \pm 0.3 \mu_{B} / \mathrm{Mn}$ for the 8-nm sample and 3.9 $\pm 0.2 \mu_{B} / \mathrm{Mn}$ for the 13-nm sample. An analysis of the polarized neutron data on both samples shows that in an individual $\mathrm{MnO}$ nanoparticle about $80 \%$ of $\mathrm{Mn}$ ions order. These results can be explained by a structure in which the monodisperse nanoparticles studied here have a core that behaves similar to the bulk with a surface layer which does not contribute significantly to the magnetic order.
\end{abstract}

DOI: 10.1103/PhysRevB.83.214418

PACS number(s): 75.30.-m, 61.05.F-, 61.46.Df

\section{INTRODUCTION}

The fundamental magnetic behavior at the nanoscale has attracted considerable attention due to potential technological applications such as magnetic data storage ${ }^{1}$ and energy-storage lithium-ion batteries. ${ }^{2}$ Because of the finite size of nanoscale magnetic materials, the large surface-to-core ratio becomes significant and, in some cases, explains the fascinating nanoscale behavior. The number of nearest neighbors for an atom at the surface is significantly reduced compared to the bulk with the consequence that the magnetic exchange decreases. Consequently, the magnetization and the transition temperature to magnetic order may be substantially reduced when compared to the bulk. Theoretical calculations have predicted this behavior. ${ }^{3-5}$ Experimentally, this behavior has been observed in both $3 d$ metals $^{6}$ and oxide samples such as $\mathrm{MnO}^{7,8}$ and $\mathrm{NiO}^{9}$ In contrast, an increase has been observed in the magnetic ordering temperature and magnetic moment in several nanoscale materials. ${ }^{10-15}$ For example, in some nanoscale $3 d$ metals, the moment is enhanced as a result of band narrowing at the surface resulting from the reduced coordination number. This behavior has been observed experimentally in iron, cobalt, and nickel clusters ${ }^{10}$ and has been supported by theoretical calculations. ${ }^{16}$ On the other hand, in ionic oxide compounds, due to the relatively localized electronic distribution, the moment is less affected by the surface and surface disorder results in a reduced average moment. ${ }^{17}$ A reduced moment and enhanced transition temperature have been observed in $\mathrm{MnO}$ particles ${ }^{11-15}$ and in the spinel ferrites $\mathrm{Ni}(\mathrm{Mn}) \mathrm{Fe}_{2} \mathrm{O}_{4} \cdot{ }^{18}$ In either case, the origin of the moment and ordering temperature enhancement or reduction is unclear and, consequently, further study of magnetic nanoparticles is important.

The classic antiferromagnetic (AFM) oxide compound $\mathrm{MnO}$ is a good candidate for studying finite size effects due to its relatively simple structure and well-studied bulk properties. Bulk MnO shows an AFM transition at $T_{N} \approx 118 \mathrm{~K}-120 \mathrm{~K}^{19-21}$ that occurs concomitantly with a rhombohedral structural distortion from the high-temperature $\mathrm{NaCl}$ structure. ${ }^{22}$ Previous studies have shown that the AFM order and structural transition of $\mathrm{MnO}$ survive into the nanoscale regime. ${ }^{11}$ The nature of the effect and its root cause remain unresolved because in some studies an enhanced $T_{N}$ has been observed for confinement particles ${ }^{11,13,23}$ and core-shell particles ${ }^{12,14}$ while a suppressed $T_{N}$ is reported in so-called ultrafine particles. ${ }^{7,8}$ In another example of $\mathrm{MnO}$ nanoparticles, only short-range AFM order is reported. ${ }^{24}$ The degree to which this difference in behavior is related to the magnetic domain size or to the surface preparation remains unclear. Hence, studies that compare multiple sizes of nanoscale $\mathrm{MnO}$ with similar surface preparations can shed light on the intrinsic magnetic behavior that results from size confinement.

In this paper we report neutron diffraction measurements on two different sizes ( 8 and $13 \mathrm{~nm}$ ) of monodisperse spherical $\mathrm{MnO}$ nanoparticles. The 8- (13)-nm sample is denoted as sample A (B) in the following discussion. Our results show that in sample A the AFM transition temperature $T_{N}$ is suppressed while in sample $\mathrm{B}, T_{N}$ is almost the same as bulk $\mathrm{MnO}$. The $\mathrm{Mn}$ magnetic moment is similar in both samples and is about $80 \%$ of the bulk value of $4.89 \mu_{B} / \mathrm{Mn}^{21}$ From polarized neutron diffraction data we estimate that the surface-to-total ratio is about $20 \%$, which appears to explain the reduction in the moment.

\section{EXPERIMENTAL DETAILS}

Monodisperse $\mathrm{MnO}$ nanoparticles were synthesized through a modification of noninjection synthetic schemes. ${ }^{25}$ An important aspect of the synthesis process is the attachment of capping ligands, which provides size control, minimizes interparticle interactions, and passivates the surface. To minimize difficulties associated with the large incoherent neutronscattering cross section of hydrogen, deuterated capping ligands were used. Approximately ten batches for each sample size were combined to produce samples large enough for 
neutron-scattering experiments $(\sim 0.5 \mathrm{~g}$ each $)$. Two distinct sizes were studied, an 8-nm sample (sample A) and a 13-nm (sample B). These particle sizes were determined from transmission electron microscopy (TEM) of $\sim 50-100$ particles per sample. As is discussed further below, a limitation of TEM is the low number of particles sampled; however, despite this limitation the TEM can provide some information concerning the polydispersity of the samples studied here. The TEM results on sample A give a particle size of $7.9 \pm 1.6 \mathrm{~nm}$ (Ref. 25) and for sample B give a particle size of $13 \pm 2 \mathrm{~nm}$ [see insets Figs. 3(a) and 3(b)]. The shape of nanoparticle samples is an important consideration. Aside from the difficulty in forming anisotropic particles of $\mathrm{MnO}$ using our methods, there are two primary lines of evidence for the production of spherical particles. The first comes from TEM images. If other shapes form, projection in two dimensions will reveal other geometries. For example, cubic nanoparticles will occasionally (for suitable orientation) reveal hexagonal shapes based on the 2D projection. Additionally, if plates are formed, these are generally transparent in TEM imaging and this was not the case here. Furthermore, if growth occurs along a particular crystallographic face, this will be revealed in X-ray and neutron diffraction patterns. Indeed, preferred growth directions will be associated with different peak widths for different reflections. In this case, no such divergence was found, consistent with the formation of isotropic (i.e., spherical) particles, in strong corroboration of our TEM results.

$\mathrm{X}$-ray diffraction with $\mathrm{Cu} K \alpha$ radiation $(\lambda=1.5406 \AA)$ and polarized and unpolarized neutron diffraction experiments were performed. The neutron diffraction experiments were performed on several instruments: the HB1A tripleaxis instrument with $\lambda=2.364 \AA$ at the High Flux Isotope Reactor (HFIR) of Oak Ridge National Laboratory; the C2 diffractometer at the Canadian Neutron Beam Centre (CNBC) in Chalk River, Canada, with neutron wavelength of $\lambda=1.3306$ and $2.3721 \AA$; the general purpose neutron polarization analysis spectrometer, D7, with $\lambda=3.073 \AA$ at the Institut Laue-Langevin in Grenoble, France; and the DNS polarized diffuse scattering instrument with $\lambda=4.74 \AA$ operated by the Jülich Centre for Neutron Science (JCNS) at the Forschungsneutronenquelle Heinz Maier-Leibnitz (FRM II), TU München, Germany.

To carefully extract the particle size as well as the magnetic domain size from a diffraction pattern, it is important to properly account for instrumental resolution. A standard run on a mixture of NIST Si 640c and annealed yttria was performed to determine the instrumental resolution of $\mathrm{C} 2$. Similarly, a $\mathrm{Y}_{3} \mathrm{Fe}_{5} \mathrm{O}_{12}$ standard was used to determine the instrumental resolution of the D7. For HB1A the instrumental resolution was obtained by comparing with a Si standard and with a sample of bulk MnO. The particle size and magnetic domain size were obtained from analysis of the (111) and $\left(\frac{1}{2} \frac{1}{2} \frac{1}{2}\right)$ (pseudocubic notation) peak width using the standard Scherrer formula ${ }^{26}$ and refinement of the diffraction pattern using FULLPROF. ${ }^{27}$ In particular, two methods were used to extract the particle size and magnetic domain size from the neutron diffraction data. In the first method, the instrumental resolution obtained as described above was convolved with the fitted Gaussian peak width. In the second method, the instrument resolution peak shape parameters were fixed and a peak broadening parameter, to account for finite size effects, was included in the FULLPROF refinements of the data. All particle sizes determined from the neutron diffraction data are reported with the instrumental resolution taken into account.

\section{RESULTS AND ANALYSIS}

\section{A. Sample A (8 nm)}

Figure 1(a) shows the X-ray diffraction pattern of sample A. The diffuse background is likely a consequence of the capping ligand. Analysis of the widths of the (111), (002), and (022) reflections using the Scherrer formula ${ }^{26}$ yields particle sizes of $9.5 \pm 0.2,9.7 \pm 0.1$, and $9.2 \pm 0.1 \mathrm{~nm}$, similar to the values determined from TEM of $7.9 \pm 1.6 \mathrm{~nm} .{ }^{25}$ Note that in contrast to the TEM data, the error bars on the particle size determined from the X-ray data (as well as the neutron data discussed
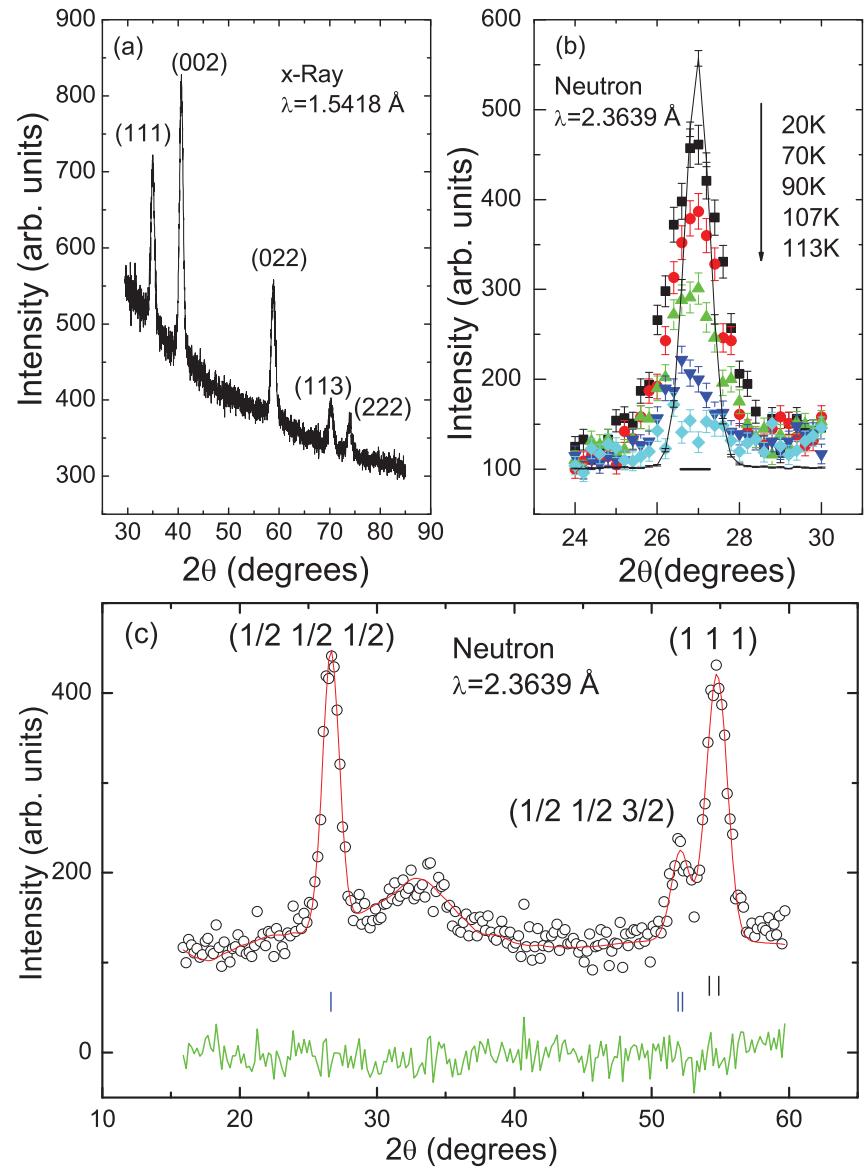

FIG. 1. (Color online) (a) X-ray diffraction pattern of $\mathrm{MnO}$ nanoparticle sample A. (b) The temperature dependence of the $\left(\frac{1}{2} \frac{1}{2} \frac{1}{2}\right)$ magnetic Bragg peak for sample A. The solid line is the scaled data for the bulk material and the horizontal bar indicates the full width at half maximum (FWHM) of the instrumental resolution. (c) Neutron diffraction data for sample A at $20 \mathrm{~K}$. The data were collected on HB1A. The broad hump between $30^{\circ}$ and $40^{\circ}$ is likely the contribution from the capping ligand and/or residual solvent. This hump is also observed in the coherent + isotope incoherent scattering channel for sample B at $Q \sim 1.5 \AA$ [see Fig. 3(c)], indicating that this scattering is of nonmagnetic origin. The solid line is the result of the Rietveld refinement. The short vertical lines indicate the nuclear Bragg peak (upper) and magnetic Bragg peak (lower). 
below) reflect a statistical error of the mean particle size and do not provide information concerning the polydispersity of the samples. In Fig. 1(c) we present neutron diffraction data collected on the triple-axis instrument HB1A with $\lambda=$ $2.3639 \AA$ at $20 \mathrm{~K}$. As shown in Fig. 1(b), the peak width of the nanoparticle sample is obviously broadened when compared to the bulk material. The particle size determined from the nuclear peak (accounting for instrumental resolution) is $9.9 \pm 0.5 \mathrm{~nm}$, in good agreement with the $\mathrm{x}$-ray values. Here we note that the amount of material used differs significantly for the different techniques. TEM measurements sample the fewest particles ( 50-100 particles) followed by x-ray diffraction $(\sim 50 \mathrm{mg})$ and, finally, neutron diffraction $(\sim 0.5 \mathrm{~g})$. Given this, we use the neutron-derived values of the particle size as being most representative of the samples measured. In a similar fashion, the effective magnetic domain size can be determined from the width of the magnetic Bragg peaks. In sample A at $20 \mathrm{~K}$ this analysis yields a magnetic domain size of $9.2 \pm 0.4 \mathrm{~nm}$. Within the experimental resolution of this measurement we are unable to determine a shift in lattice constant compared to the bulk; however, the $\mathrm{x}$-ray data (higher resolution) on samples from this batch indicate a somewhat smaller lattice constant [4.439(1) ̊]].

In Fig. 2, we present the temperature dependence of the magnetic moment for both bulk $\mathrm{MnO}$ and sample A. No significant thermal hysteresis was observed in either the bulk or nanoparticle material, indicating that the samples were in thermal equilibrium. The moment was obtained by scaling the integrated intensities of magnetic and structural Bragg reflections, assuming a moment of $4.89 \mu_{B} / \mathrm{Mn}^{21}$ for bulk $\mathrm{MnO}$. This results in a low-temperature magnetic moment for sample $\mathrm{A}$ of $4.07 \pm 0.11 \mu_{B} / \mathrm{Mn}$ at $20 \mathrm{~K}$. Note that this analysis assumes the magnetic structure of the nanoparticle and the bulk is the same aside from the magnitude of the ordered magnetic moment-justification of this assumption is given below. From Fig. 2, we can clearly see that not only is the magnetic moment suppressed in the nanoparticle sample relative to the bulk, but that the AFM transition temperature $T_{N}$ decreases from $118.7 \mathrm{~K}$ in bulk to $113.6 \mathrm{~K}$ in sample A. A

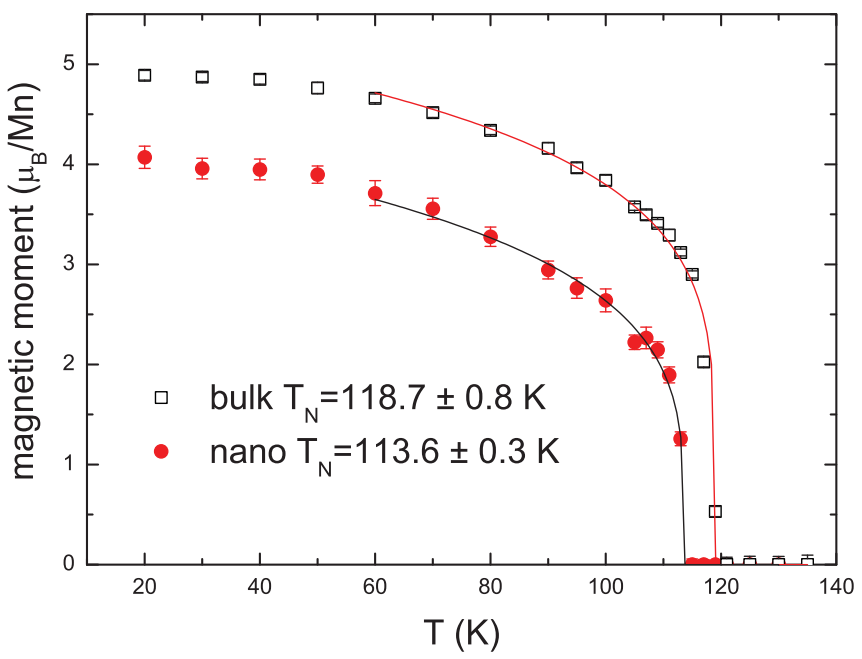

FIG. 2. (Color online) The temperature-dependent magnetic moment of the $\mathrm{MnO}$ bulk and nanoparticles of sample A. The solid lines are guides for the eye. similar suppression of $T_{N}$ has been reported in ultrafine $\mathrm{MnO}$ particles with sizes in the range of $\sim 3.7$ to $\sim 5.4 \mathrm{~nm}^{7,8}$

To provide further insight into the above results, models of both the crystal and the magnetic structure were fit to the neutron diffraction data of sample $A$ and a sample of bulk $\mathrm{MnO}$ at $20 \mathrm{~K}$, through Rietveld refinement using FULLPROF to determine the magnetic moment, particle size, and domain size. A model including the rhombohedral structural distortion $(R \overline{3} m)^{28}$ was used for the refinement and the bulk diffraction pattern was used to specify the instrument resolution function for the sample A refinement. Fitting the bulk neutron-scattering data yields a magnetic moment of $4.8 \pm 0.3 \mu_{B} / \mathrm{Mn}$, consistent with the previously reported value. $^{21}$ The resulting refinement for sample $\mathrm{A}$ is indicated by the solid line in Fig. 1(c). The results yield a magnetic moment of $4.2 \pm 0.3 \mu_{B} / \mathrm{Mn}$, a particle size of $10.3 \pm 0.5 \mathrm{~nm}$ and a magnetic domain size of $9.1 \pm 0.7 \mathrm{~nm}$, consistent with the analysis above using the Scherrer formula. Here we note that the magnetic domain size is almost as large as the nuclear particle size $(\sim 1.5$ magnetic domains per nanoparticle); thus, the dominant magnetic behavior appears to come from a single magnetic domain per nanoparticle. This point is discussed further below.

\section{B. Sample B (13 nm)}

In Fig. 3(a) room temperature diffraction data are presented for sample B. Analyzing the (111) nuclear reflection yields a particle size of $13.1 \pm 0.7 \mathrm{~nm}$. Figure 3(b) shows the diffraction pattern at $4 \mathrm{~K}$. A similar analysis of the width of the magnetic peak $\left(\frac{1}{2} \frac{1}{2} \frac{1}{2}\right)$ gives a magnetic domain size of $10.0 \pm 0.2 \mathrm{~nm}$. Polarized neutron diffraction data allows for the unambiguous separation of the magnetic from the nonmagnetic scattering. In Fig. 3(c) we present polarized neutron diffraction data separated into various components of the neutron scattering cross section. Here the single peak analysis yields a magnetic domain size of $9.7 \pm 0.4 \mathrm{~nm}$ and a particle size of $12.6 \pm 0.8 \mathrm{~nm}$. Thus, in this case, there appears to be the possibility of two magnetic domains per nanoparticle.

To thoroughly analyze the diffraction data, FULLPROF was used for the refinement of the entire diffraction pattern. The solid lines in Figs. 3(a) and 3(b) are the results of the refinement. As in the bulk material, at a temperature above the AFM transition the diffraction pattern can be well described by a $\mathrm{NaCl}$ structure $(F m \overline{3} m)$, while below the transition temperature a structural model including a rhombohedral distortion $(R \overline{3} m)$ best describes the data.

In Fig. 4 we present the results of structural refinements as a function of temperature for sample B. The temperaturedependent lattice parameter is shown in Fig. 4(a); the stars are the results for the high-temperature $\mathrm{NaCl}$ phase. The temperature dependence of the magnetic moment is shown in Fig. 4(b). This allows us to extract an AFM transition temperature of $120 \pm 0.3 \mathrm{~K}$, comparable, or perhaps slightly larger than that of the bulk material. At $4 \mathrm{~K}$ the magnetic moment saturates at a value of $3.9 \pm 0.2 \mu_{B} / \mathrm{Mn}$, which is similar to the previous reported value for $\mathrm{MnO}$ nanoparticles embedded in porous glass. ${ }^{11}$ Figure 4(c) shows the temperature dependence of the rhombohedral distortion angle, $\Delta \alpha$. $\Delta \alpha$ increases with decreasing temperature saturating at a 


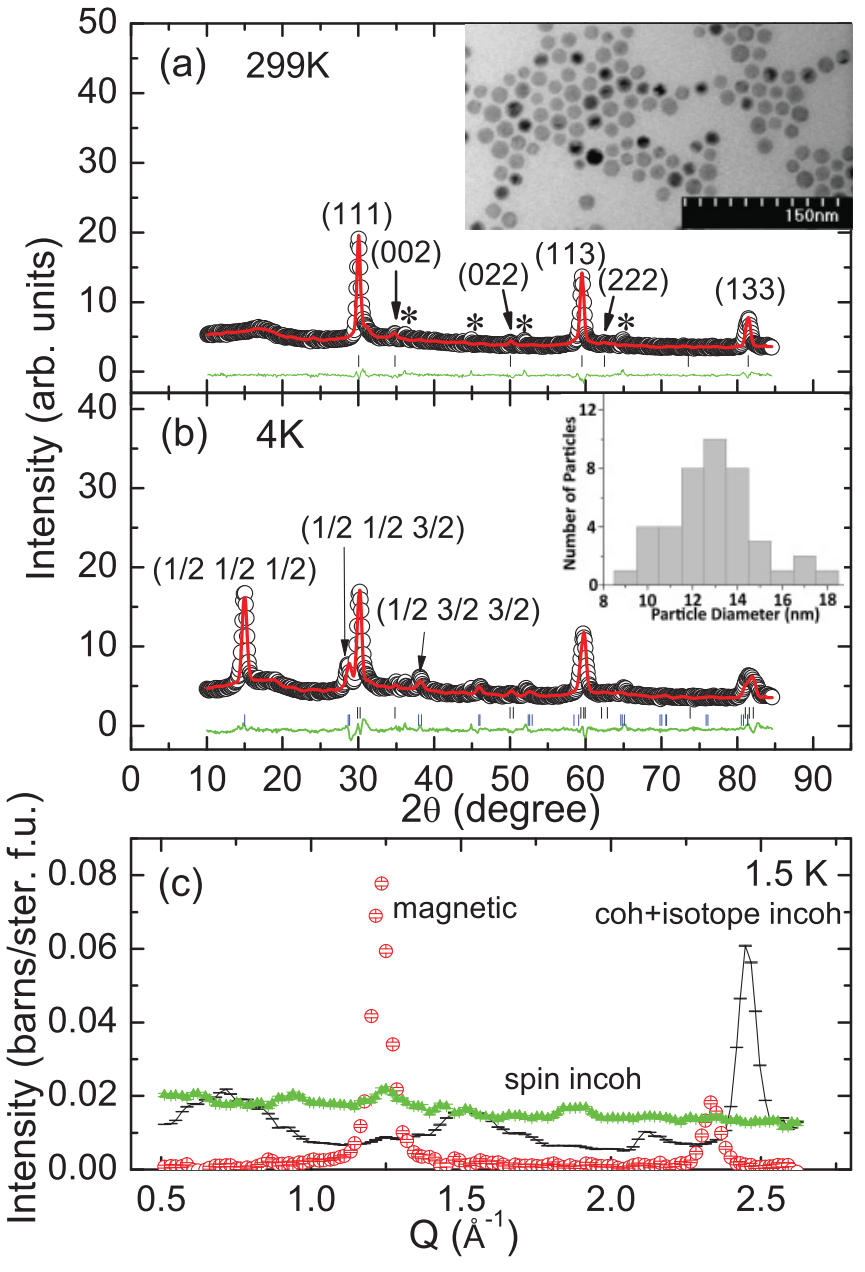

FIG. 3. (Color online) The observed (open circles) and calculated (solid lines) neutron diffraction data for nanoparticle sample B at room temperature (a) and $4 \mathrm{~K}$ (b). The weak peaks indicated by the star in (a) are due to a $\mathrm{MnO}_{2}$ impurity. The data were collected on $\mathrm{C} 2$. The short vertical lines indicates the nuclear (upper) and magnetic (lower) Bragg Peak position. (c) The separated polarized neutron diffraction data. The intensity is presented in units of barns per steradian per formula unit. The data were collected on D7. The solid line is the sum of the coherent and isotope incoherent, the solid triangle is nuclear spin incoherent scattering, and the open circle is the magnetic scattering. The inset in (a) is the TEM image. The inset in (b) is the particle size distribution.

value of $0.44 \pm 0.01^{\circ}$. This value is slightly smaller than both the nanoparticles embedded in porous glass ${ }^{11}$ and the bulk ${ }^{20}$ where $\Delta \alpha \sim 0.6^{\circ}$. However, a smaller value of about $0.43^{\circ}$ has also been reported in bulk. ${ }^{28}$ The temperature dependence of the distortion angle indicates a structural transition temperature of $120 \pm 0.3 \mathrm{~K}$, which is the same as the $T_{N}$ obtained from the evolution of the magnetic order parameter.

The temperature evolution of the $\left(\frac{1}{2} \frac{1}{2} \frac{1}{2}\right)$ magnetic Bragg peak is displayed in the insets of Figs. 5(a) and Fig. 5(b), respectively. In Fig. 5(a) the temperature dependence of the full width at half maximum (FWHM) of the $\left(\frac{1}{2} \frac{1}{2} \frac{1}{2}\right)$ peak is shown for sample B. Above $T_{N}$, there is no obvious signal at $\left(\frac{1}{2} \frac{1}{2} \frac{1}{2}\right)$; we include the (111) nuclear peak width for comparison (solid symbol). The magnetic peak width shows a drastic change at

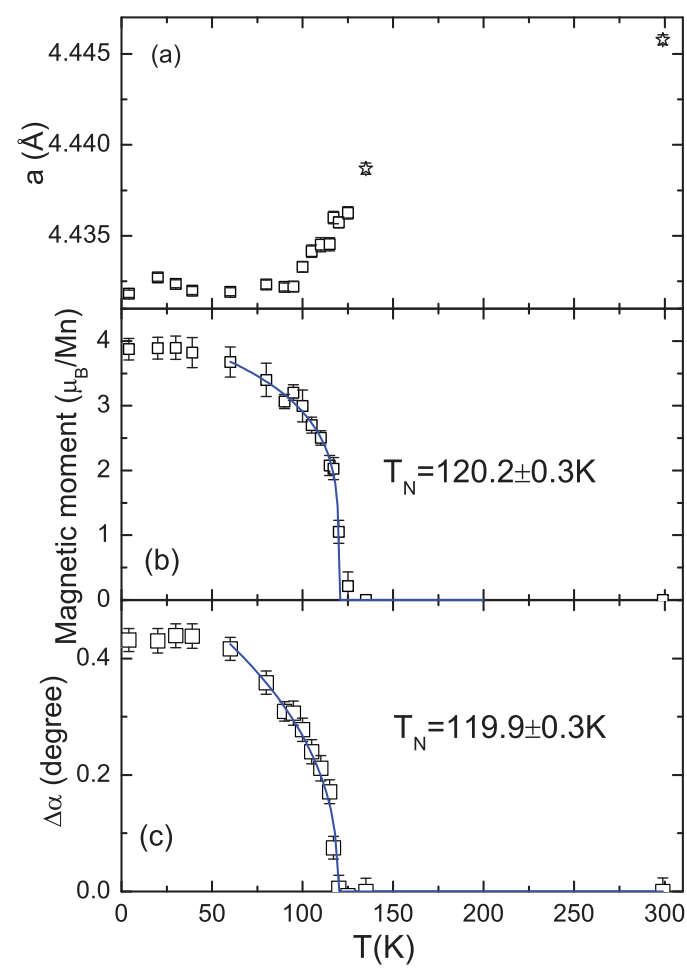

FIG. 4. (Color online) Temperature dependence of the lattice parameter (a), magnetic moment (b), and the structure distortion angle, $\Delta \alpha$ (c), for sample $\mathrm{B}$. The results are obtained through refinement of models of the crystal and magnetic structure to the neutron scattering data from $\mathrm{C} 2$ as described in the text. The solid lines in (b) and (c) are guides for the eye.

$T_{N}$ and becomes progressively narrower below $T_{N}$, indicating that the magnetic domains emerge and grow progressively with decreasing temperature. The temperature dependence of the refined average domain diameter is shown in Fig. 5(b). For comparison, above $T_{N}$, the average particle size is plotted (solid symbols). A similar domain size is determined from the polarized neutron scattering data. The results obtained from both C2 (unpolarized) and D7 (polarized) are plotted together in Fig. 5(b) for comparison.

\section{DISCUSSION}

The summary of the particle and magnetic parameters are listed in Table I. Both samples exhibit a suppressed magnetic moment compared to bulk $\mathrm{MnO}$. This behavior is also observed in confinement geometry and core-shell $\mathrm{MnO}$ particles ${ }^{11,13,14}$ and is believed to be the result of disordered spins on the surface of the nanoparticles. ${ }^{11,17}$ However, whether the actual moment for the $\mathrm{Mn}$ site is the same as in the bulk has not been directly determined yet. To test this, polarized neutron scattering data which are sensitive to both the ordered and the disordered moments is required. Previous polarized neutron scattering data on average 7-nm confinement geometry nanoparticles indicated that only $40 \%$ of the $\mathrm{Mn}$ atoms were ordered. ${ }^{23}$ However, the average magnetic moment is not reported. In the polarized data presented here on sample B at $1.5 \mathrm{~K}$, from the peak-to-diffuse scattering ratio we can estimate that about $80 \%-85 \%$ of the $\mathrm{Mn}$ atoms in a nanoparticle are 


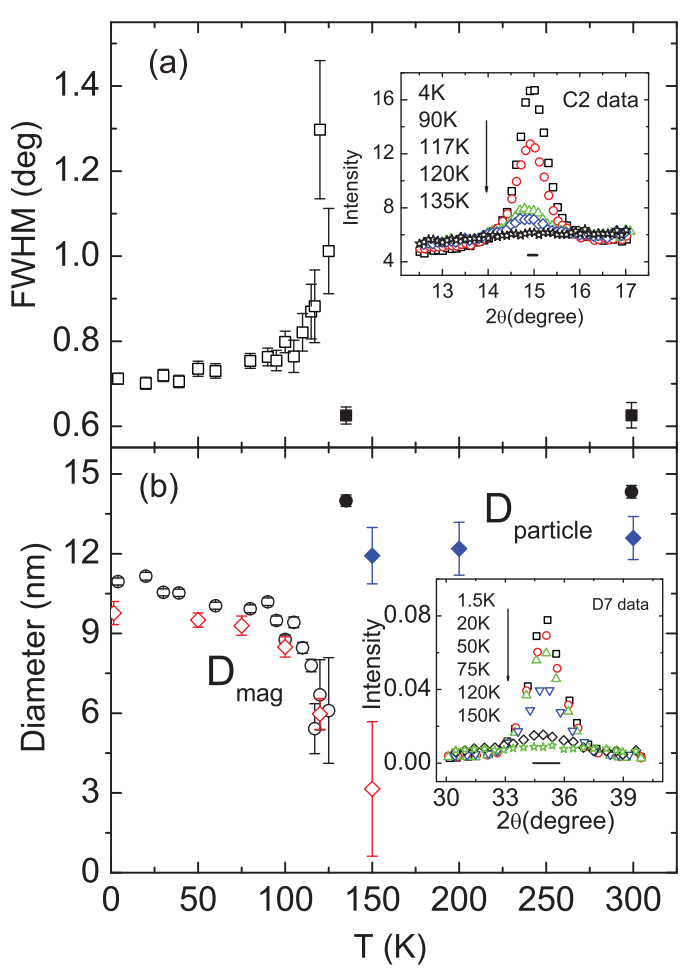

FIG. 5. (Color online) (a) $\left(\frac{1}{2} \frac{1}{2} \frac{1}{2}\right)$ magnetic peak width (open symbols) and the (111) nuclear peak width (solid symbols) for sample B. Inset is the $\left(\frac{1}{2} \frac{1}{2} \frac{1}{2}\right)$ peak at different temperatures. The data were collected on the $\mathrm{C} 2$ instrument. (b) The temperature dependent magnetic domain size (open symbols) and particle size (solid symbols) for sample B. Circles are unpolarized results from $\mathrm{C} 2$ and diamonds are polarized results from D7. Inset is the evolution of the $\left(\frac{1}{2} \frac{1}{2} \frac{1}{2}\right)$ magnetic peak with temperature from the D7 polarized neutron scattering data. Horizontal bars in the insets represent the instrumental resolution.

magnetically ordered. Assuming the actual magnetic moment of $\mathrm{Mn}$ atoms in the nanoparticle is identical to the bulk value and scaling by the percent of the particle that actually participates in magnetically ordered state, the expected average moment in this sample would be 3.9-4.1 $\mu_{B} / \mathrm{Mn}$. This is consistent with the values of the magnetic moment derived from the neutron scattering data reported in Table I. In sample $\mathrm{A}$, the average magnetic moment is about $85 \%$ of the bulk material. The polarized diffraction results collected using DNS (not shown) show that about $75 \%-80 \% \mathrm{Mn}$ atoms order at 3 $\mathrm{K}$, again, implying that the ordered moment of $\mathrm{Mn}$ site is similar to that of the bulk. One possible explanation for this observation is that the nanoparticles studied here contain a core that acts like bulk $\mathrm{MnO}$ and a shell with random spins.

The nature of the surface spins has been discussed frequently in the literature. In particular, magnetic susceptibility measurements have been interpreted in terms of weak ferromagnetism and related superparamagnetic like behavior of uncompensated surface spins. ${ }^{29-32}$ However, the size dependence of the superparamagnetic behavior appears to be inversely related to particle size in contrast to the direct relationship expected for a superparamagnetic particle. Morales et al. ${ }^{29}$ have proposed that the Mn residing on the surface is subject to a noncubic crystalline electric field and
TABLE I. Summary of the particle and magnetic parameters obtained through x-ray, TEM, and neutron scattering analysis. $D_{\text {particle }}$ and $D_{\mathrm{mag}}$ are the diameter of particles and magnetic domain size respectively. Error bars in the x-ray and neutron diffraction results represent statistical standard deviation $\pm \sigma$ of the mean particle size while the TEM results represent the standard deviation of the size distribution.

\begin{tabular}{lcc}
\hline \hline Samples & $\mathrm{A}$ & $\mathrm{B}$ \\
\hline$D_{\text {particle }}(\mathrm{nm})$ & $10.3 \pm 0.5(\mathrm{HB} 1 \mathrm{~A})$ & $14.0 \pm 0.2(\mathrm{C} 2)$ \\
& $9.5 \pm 0.2(\mathrm{x}-\mathrm{ray})$ & $12.6 \pm 0.8(\mathrm{D} 7)$ \\
& $7.9 \pm 1.6(\mathrm{TEM})$ & $13.0 \pm 2.0(\mathrm{TEM})$ \\
$D_{\mathrm{mag}}(\mathrm{nm})$ & $9.1 \pm 0.7(\mathrm{HB} 1 \mathrm{~A})$ & $10.9 \pm 0.1(\mathrm{C} 2)$ \\
& & $9.7 \pm 0.4(\mathrm{D} 7)$ \\
$T_{N}(\mathrm{~K})$ & $113.6 \pm 0.2(\mathrm{HB} 1 \mathrm{~A})$ & $120.2 \pm 0.3(\mathrm{C} 2)$ \\
Moment $\left(\mu_{B} / \mathrm{Mn}\right)$ & $4.2 \pm 0.3(\mathrm{HB} 1 \mathrm{~A})$ & $3.9 \pm 0.2(\mathrm{C} 2)$ \\
& & $3.9 \pm 0.2(\mathrm{D} 7)$ \\
\hline \hline
\end{tabular}

this provides an additional source of anisotropy effecting the superparamagnetic behavior. In this lower-symmetry crystalfield environment, a low-spin configuration is energetically favorable, resulting in a moment of $\sim 1 \mu_{B}$ on the surface Mn atoms. Unfortunately, the neutron-scattering data here cannot contribute significantly to the discussion concerning the nature of the surface spins. The diffuse magnetic scattering in the polarized neutron scattering data [Fig. 3(c)] shows a weak magnetic diffuse component that suggests the presence of disordered spins, but we stress though that the measurement itself provides no information on whether these spins are on the surface. Moreover, if there were significant ferromagnetic moments we would expect depolarization of the neutron beam. No obvious depolarization of the neutron beam was observed, but it is difficult make a quantitative estimate on a limit of the a ferromagnetic moment size without further systematic measurements.

A comparison of $T_{N}$ for bulk $\mathrm{MnO}$ to that of sample A (8 $\mathrm{nm}$ ), shows that $T_{N}$ is suppressed by about $4 \%$ compared to the bulk value. On the other hand, sample B $(13 \mathrm{~nm})$, exhibits a $T_{N}$ comparable to the bulk. Suppression of $T_{N}$ is also observed in ultrafine $\mathrm{MnO}$ particles, ${ }^{7}$ as well as in other nanoscale AFM compounds such as $\mathrm{NiO}$ and $\mathrm{CoO}$ thin films, ${ }^{33,34}$ and $\mathrm{NiO}$ disk shaped nanoparticles. ${ }^{9}$ A mean field approach with finite size effects for the magnetic transition has been used for the explanation of the suppression of $T_{N}$. In this theory, the transition temperature is suppressed when the sample size and/or thickness decreases to small value. For $\mathrm{MnO}$ a prism model of ultrafine particles, the calculations predict $T_{N}$ will be suppressed at small size due to the decrease of the average coupling constant which is induced by the decrease of coordination number on the surface. ${ }^{7}$ Similar arguments are likely to apply to the nanoparticles studied here and thus $T_{N}$ suppression is likely due to decreases in the coordination number and the average coupling constant. In sample B, $T_{N}$ remains the same as that seen in the bulk material, suggesting that size confinement effects in $\mathrm{MnO}$ nanoparticles appear at only relatively small sizes. In apparent contradiction to the preceding arguments, an enhanced $T_{N}$ is reported in confined geometry nanoparticles. ${ }^{11,13,14,23}$ For these nanoparticle 
systems the wormlike particle morphology ${ }^{11,13,23}$ and the surface interface of glass ${ }^{11,13,23}$ or another magnetic species ${ }^{14}$ are the likely cause of the differences in behavior with those studied here. As mentioned above, the coordination number on the surface affects the transition temperature and by adjusting the coordination number $T_{N}$ can be increased or decreased. ${ }^{35}$

The effect of size confinement on the order of the magnetic and structural phase transition is another interesting issue in nanoscale $\mathrm{MnO}$. It has been suggested in the confined geometry $\mathrm{MnO}$ nanoparticles that the AFM transition is a continuous phase transition. ${ }^{11,13}$ In these samples the critical exponent $\beta$ describing the ordered magnetic moment, $M(T) \sim\left(1-T / T_{N}\right)^{\beta}$, ranges from 0.3 up to almost the mean field value of 0.5 . These values are in reasonable agreement with Monte Carlo simulations for the finite-size Ising model and Heisenberg model, where $\beta$ is 0.3258 and 0.3616 , respectively. ${ }^{36}$ In the reduced temperature range of $1-T / T_{N}=0.02-0.5$, fitting our temperature-dependent moment to a power law of $\left(1-T / T_{N}\right)^{\beta}$ yields a much smaller $\beta$ of $0.24 \pm 0.02$ for sample $\mathrm{A}$ and $0.22 \pm 0.02$ for sample B. This could be interpreted as lower-dimensional critical behavior but is more likely the result of a discontinuous phase transition.
In conclusion, we have studied two different sizes of monodisperse $\mathrm{MnO}$ nanoparticles using unpolarized and polarized neutron diffraction. Both the magnetic and the crystal structure are similar to the bulk, but with substantially reduced average magnetic moment. Moreover, the results show that in the 8-nm sample the AFM transition temperature $T_{N}$ is suppressed while in the $13-\mathrm{nm}$ sample, $T_{N}$ is comparable to that seen in the bulk. The suppression of $T_{N}$ is attributed to the exchange coupling reduction induced by the finite size effect. The observations presented here are consistent with the core of the $\mathrm{MnO}$ nanoparticles behaving much like the bulk with a disordered surface layer.

\section{ACKNOWLEDGMENTS}

We acknowledge useful discussions with J. Musfeldt and I. Swainson. Research Work at ORNL was sponsored by the Laboratory Directed Research and Development Program of ORNL, and was supported by the Scientific User Facilities Division Office of Basic Energy Sciences, DOE. A portion of this research was conducted at the Center for Nanophase Materials Sciences, which is sponsored at ORNL by the Office of Basic Energy Sciences, US DOE.
*Current address: Department of Chemical Engineering, University of Missouri, Columbia, MO 65211, USA.

${ }^{\dagger}$ Current address: Department of Chemistry, University of Missouri, Columbia, MO 65211, USA.

Current address: European Spallation Source ESS AB, Stora Algatan 4, SE-221 00 Lund, Sweden.

${ }^{1}$ D. U. Lee, T. H. Lee, E. K. Kim, J. W. Shin, and W. J. Cho, AIP Conf. Proc.: 1199, 509 (2009), Physics of Semiconductors: 29th International Conference on the Physics of Semiconductors; C. Egami, N. Nishimura, and T. Okawa, Optics Express 18, 15901 (2010).

${ }^{2}$ J. Liu and Q. M. Pan, Electrochem. Solid-State Lett. 13, A139 (2010); J. Yang, M. Winter, and J. O. Besenhard, Solid State Ionics 90, 281 (1996).

${ }^{3}$ J. Merikoski, J. Timonen, M. Manninen, and P. Jena, Phys. Rev. Lett. 66, 938 (1991).

${ }^{4}$ P. V. Hendriksen, S. Linderoth, and P. A. Lindgard, Phys. Rev. B 48, 7259 (1993).

${ }^{5}$ J. L. Morán-López, K. H. Bennemann, J. M. MontejanoCarrizales, and F. Aguilera-Granja, Solid State Commun. 88, 101 (1993).

${ }^{6}$ W. A. de Heer, P. Milani, and A. Chatelain, Phys. Rev. Lett. 65, 488 (1990).

${ }^{7}$ S. Sako and K. Ohshima, J. Phys. Soc. Jpn. 64, 944 (1995).

${ }^{8}$ S. Sako, Y. Umemura, K. Ohshima, M. Sakai, and S. Bandow, J. Phys. Soc. Jpn. 65, 280 (1996).

${ }^{9}$ S. N. Klausen, P.-A. Lindgård, K. Lefmann, F. Bødker, and S. Mørup, Phys. Status Solidi A 189, 1039 (2002).

${ }^{10}$ I. M. L. Billas, A. Châtelain, and W. A. de Heer, Science 265, 1682 (1994); I. M. L. Billas, A. Chatelain, and W. A. de Heer, J. Magn. Magn. Mater. 168, 64 (1997).
${ }^{11}$ I. V. Golosovsky, I. Mirebeau, G. André, D. A. Kurdyukov, Yu. A. Kumzerov, and S. B. Vakhrushev, Phys. Rev. Lett. 86, 5783 (2001).

${ }^{12}$ I. V. Golosovsky, G. Salazar-Alvarez, A. López-Ortega, M. A. González, J. Sort, M. Estrader, S. Surinach, M. D. Baró, and J. Nogués, Phys. Rev. Lett. 102, 247201 (2009).

${ }^{13}$ I. V. Golosovsky, I. Mirebeau, V. P. Sakhnenko, D. A. Kurdyukov, and Y. A. Kumzerov, Phys. Rev. B 72, 144409 (2005).

${ }^{14}$ A. López-Ortega, A. Tobia, E. Winkler, I. V. Golosovsky, G. Salazar-Alvarez, S. Estradé, M. Estrader, J. Sort, M. A. González, A. Surinach, J. Arbiol, F. Peiró, R. D. Zysler, M. D. Baró, and J. Nogués, J. Am. Chem. Soc. 132, 9398 (2010).

${ }^{15}$ J. W. Taylor, J. A. Duffy, C. S. Steer, and C. Shentonn-Taylor, J. Magn. Magn. Mater. 310, 2327 (2007).

${ }^{16}$ G. M. Pastor, J. Dorantes-Dávila, and K. H. Bennemann, Phys. Rev. B 40, 7642 (1989).

${ }^{17}$ R. H. Kodama, J. Magn. Magn. Mater. 200, 359 (1999).

${ }^{18}$ C. N. Chinnasamy, A. Narayanasamy, N. Ponpandian, R. J. Joseyphus, B. Jeydevan, K. Tohji, and K. Chattopadhyay, J. Magn. Magn. Mater. 238, 281 (2002); C. N. Chinnasamy, A. Yang, A. D. Yoon, K. Hsu, M. D. Shultz, E. E. Carpenter, S. Mukerjee, C. Vittoria, and V. G. Harris, J. Appl. Phys. 101, 09M509 (2007).

${ }^{19}$ C. G. Shull, W. A. Strauser, and E. O. Wollan, Phys. Rev. 83, 333 (1951).

${ }^{20}$ B. Morosin, Phys. Rev. B 1, 236 (1970).

${ }^{21}$ M. Bonfante, B. Hennion, F. Moussa, and G. Pepy, Solid State Commun. 10, 553 (1972).

${ }^{22}$ N. C. Tombs and H. P. Rooksby, Nature (London) 165, 442 (1950).

${ }^{23}$ M. Feygenson, W. Schweika, A. Ioffe, S. B. Vakhrushev, and Thomas Brückel, Phys. Rev. B 81, 064423 (2010). 
${ }^{24}$ T. Chatterji, Y. Su, G. N. Iles, Y.-C. Lee, A. P. Khandhar, and K. M. Krishnan, J. Magn. Magn. Mater. 322, 333 (2010).

${ }^{25}$ Qi-C. Sun, X. Xu, S. N. Baker, A. D. Christianson, and J. L. Musfeldt, Chem. Mater. 23, 2956 (2011).

${ }^{26}$ A. L. Patterson, Phys. Rev. 56, 978 (1939).

${ }^{27}$ J. Rodriguez-Carvajal, Physica B 192, 55 (1993).

${ }^{28}$ W. L. Roth, Phys. Rev. 110, 1333 (1958).

${ }^{29}$ M. A. Morales, R. Skomski, S. Fritz, G. Shelburne, J. E. Shield, Ming Yin, S. O’Brien, and D. L. Leslie-Pelecky, Phys. Rev. B 75, 134423 (2007).

${ }^{30}$ M. Ghosh, K. Biswas, A. Sundaresan, and C. N. R. Rao, J. Mater. Chem. 16, 106 (2006).
${ }^{31}$ G. H. Lee, S. H. Huh, J. W. Jeong, B. J. Choi, S. H. Kim, and H.-C. Ri, J. Am. Chem. Soc. 124, 12094 (2002).

${ }^{32}$ W. S. Seo, H. H. Jo, K. Lee, B. Kim, S. J. Oh, and J. T. Park, Angew. Chem., Int. Ed. 43, 1115 (2004).

${ }^{33}$ D. Alders, L. H. Tjeng, F. C. Vooht, T. Hibma, G. A. Sawatzky, C. T. Chen, J. Vogel, M. Sacchi, and S. Iacobucci, Phys. Rev. B 57, 11623 (1998).

${ }^{34}$ E. N. Abarra, K. Takano, F. Hellman, and A. E. Berkowitz, Phys. Rev. Lett. 77, 3451 (1996).

${ }^{35}$ J. M. Wesselinowa, J. Magn. Magn. Mater. 322, 234 (2010).

${ }^{36}$ D. P. Landau, J. Magn. Magn. Mater. 200, 231 (1999). 